\title{
Nuevas tecnologías: Puentes de comunicación en el trastorno del espectro autista (TEA)
}

\section{New technologies: communication bridges in autism spectrum disorders (ASD)}

\author{
Guido Guzmán \\ Laboratorio de Aprendizaje Biológico y Artificial, Departamento de Ingeniería Biomédica, Instituto de Ciencias Básicas y \\ Medicina Experimental, Instituto Universitario Hospital Italiano de Buenos Aires, Buenos Aires, Argentina \\ Natalia Putrino \\ Facultad de Psicología, Universidad de Buenos Aires, Buenos Aires, Argentina. \\ Centro Interdisciplinario de Investigaciones en Psicología Matemática y Experimental (CIIPME) - Unidad Ejecutora del \\ Consejo Nacional de Investigaciones Científicas y Técnicas (CONICET) \\ Facultad de Psicología, Universidad Abierta Interamericana

\section{Felipe Martínez} \\ Facultad de Psicología, Universidad de Buenos Aires, Buenos Aires, Argentina.
}

\section{Nicolás Quiroz}

Laboratorio de Aprendizaje Biológico y Artificial, Departamento de Ingeniería Biomédica, Instituto de Ciencias Básicas y Medicina Experimental, Instituto Universitario Hospital Italiano de Buenos Aires, Buenos Aires, Argentina

Recepcionado (1 de Septiembre 2016) Aceptado (21 de julio 2017)

\section{Correspondencia:}

Correo electrónico de contacto: guido.guzman@hospitalitaliano.org.ar, Laboratorio de Aprendizaje Biológico y Artificial, Departamento de Ingeniería Biomédica, Instituto de Ciencias Básicas y Medicina Experimental, Instituto Universitario Hospital Italiano de Buenos Aires. Potosí 42404 to Piso, CP 1199ACL, Ciudad Autónoma de Buenos Aires, Argentina.

Agradecimientos: Los autores agradecemos el apoyo y colaboración especial del Dr. Angel Elgier, Director de la carrera de Psicología de la Universidad Abierta Interamericana e Investigador CONICET, y al Instituto de Ciencias Básicas y Medicina Experimental del Instituto Universitario del Hospital Italiano, la Dra Valeria Burgos del Depto. de Ing. Biomédica y los directivos, Dr. Marcelo Figari, Dra. Karin Kopitowski y Lic. Mariana Barbich por brindarnos su apoyo para realizar las investigaciones y desarrollos en tecnología y TEA. También la colaboración del personal del Servicio de Salud Mental Pediátrica del Hospital Italiano de Buenos Aires. 


\title{
Resumen
}

Los trastornos del espectro autista (TEA) son alteraciones del neurodesarrollo caracterizados por dificultades en interacción social, con principal hincapié en la comunicación, sea esta verbal y no verbal. La investigación y desarrollo aplicada en TEA se centra en hallar estrategias terapéuticas adecuadas y que sean focalizadas en el individuo, valiéndose de todos los recursos que estén al alcance (interfaces virtuales, realidad virtual, entornos 3D, robótica, etc).

Así, el uso de tecnologías para mejorar y estimular particularmente la comunicación de los niños con TEA ha aumentado en los últimos tiempos de manera exponencial. Estas herramientas en contextos terapéuticos posibilitan una generalización del comportamiento hacia contextos naturales del niño. Por lo tanto, el uso de un software personalizado en dispositivos móviles permitirá que se avance con su tratamiento fuera del ámbito clínico pudiendo utilizarlo en su casa/escuela para comunicarse con sus vínculos sociales cercanos. Palabras clave: Autismo, comunicación, tecnología, comunicación alternativa y aumentada, Apptismo.

\begin{abstract}
Autism Spectrum Disorder (ASD) refers to neurodevelopmental problems characterized by difficulties in social interaction, especially verbal or nonverbal communication. Research on ASD focuses in finding appropriate solutions for a patient, using all available resources (virtual interfaces, virtual reality, 3D environments, robotics, etc.) in order to develop therapeutic strategies.

Therefore, the use of technologies to improve and stimulate communication, particularly in children with ASD, has increased exponentially in recent years. When used in therapeutic contexts, these tools allow a generalization of a child's behavior towards a natural context. Hence, the use of customized softwares for mobile devices will allow progress in treating ASD beyond a clinical setting and may be used at home/school to communicate with their close social peers.

Key words: Autism, communication, technology, Augmentative and Alternative Communication, Apptismo.
\end{abstract}

\section{Introducción}

Este artículo realiza una conceptualización acerca del Trastorno del Espectro Autista (TEA), las terapias validadas, para luego continuar con los estudios sobre diversas tecnologías aplicadas y la importancia de su uso en un ambiente terapéutico. Finalmente se comparte la experiencia de un desarrollo teniendo en cuenta las consideraciones pertinentes para ser aplicadas en personas con TEA.

\section{La evolución de la clasificación del TEA}

Desde hace aproximadamente dos décadas, se ha comenzado a denominar TEA, en lugar de Autismo Infantil Precoz (Kanner, 1943), a aquellos que afectan a sujetos que comparten manifestaciones conductuales y cognitivas en común, pero en distintos grados, $\mathrm{y}$, esencialmente, con una condición/factor cerebral particular de base. Es por este último motivo que están incluidos en los Trastornos Generalizados del Desarrollo (Manual Diagnóstico y Estadístico de los Trastornos Mentales: DSM-IV, American Psychiatric Association 1994), como Trastorno Autista, contra poniéndose al factor ambiental/familiar que durante muchos años se creyó el preponderante para su aparición y mantenimiento (Baron-Cohen, 2008; Feinstein 2010).

La descripción de Kanner sobre el trastorno autista, además de ubicarlo como un trastorno surgido "desde el nacimiento o dentro de los primeros 30 meses de edad"; aludía a: carencia de contacto afectivo hacia otros; apego excesivo a rutinas, que a veces incluyen rareza; anomalía en el habla o mutismo; fascinación por ciertos objetos y destreza para su manipulación; y nivel superior en habilidades visoespaciales y de memoria.

El descubrimiento de Hans Asperger (1944) de personas que presentaban dichas características pero en otro grado, a las que agrupó bajo el nombre "Síndrome de Asperger", comenzó 
a dar luz sobre la posibilidad de un espectro de síntomas de misma base, más que de una especificidad. El síndrome de Asperger mostraba lo siguiente: aproximación social inadecuada e ingenua; intereses restringidos a determinadas materias u objetos; correcta gramática y vocabulario, pero pobre uso de éstas en conversación; falta de sentido común; déficit en coordinación motriz; dificultades de aprendizaje en una o dos áreas, y opcional nivel superior en otras (BaronCohen, 2008; Feinstein 2010).

Esta caracterización arrojaba similitudes con la de Kanner sobre autismo, pero era imposible tomarlas como idénticas debido a sus claras diferencias en grados, en especial, en el área de lenguaje (cuya subárea perjudicada en este caso era la pragmática/conversacional más que la fonológica, morfológica y/o semántica como en otras personas autistas) $\mathrm{y}$ de relaciones sociales (que en las personas con Asperger se dan, sólo que con falta de "sentido común" o entendimiento de "códigos compartidos", más que un aislamiento o desinterés más pronunciado como en demás personas autistas). Es por esto que se hacía cada vez más necesario un cambio en cómo considerar a las características presentes en los sujetos autistas, distintas pero con una base común; y las investigaciones de distintos especialistas en la década del 70 y 80 , fueron sirviendo de apoyo para que en los nuevos manuales de clasificación y en las consideraciones de los nuevos estudios del tema se hablará de "espectro autista" como en el DSM-5 (Baron Cohen, 2008; Feinstein 2010, American Psychiatric Association 2013).

La base común de los síntomas, actualmente, queda configurada de esta manera ("tríada de Wing"; Wing, 1996): déficits en relaciones sociales, en comunicación, y en flexibilidad de intereses e imaginación. Sobre esta base yace el espectro autista (dentro, el autismo de Kanner y el Asperger), que la dota de gradualidad y de no exclusividad de presentación de todos los síntomas agrupados en los tres grandes déficits mencionados. A estas características de los sujetos dentro del espectro autista, se pueden sumar unas no menos importantes, más “cognitivas", enalteciendo aún más la importancia de usar un espectro y no una clasificación específica o dicotómica para el diagnóstico del autismo: Dificultad para agrupar la información pasada y a raíz de este procesamiento, anticipar el futuro; déficit en clasificar las experiencias en tiempos (pasado, presente, futuro) y en espacios; dificultad al momento de seleccionar la información principal de la secundaria que le llega, de darle una "coherencia central" (Frith, 1992) o sentido; pobreza en detectar emociones o necesidades en otra persona, es decir, en la capacidad de la "Teoría de la mente"(Baron-Cohen, 2001; Juillerat, Cornejo, Castillo y Chaigneau, 2015), y un sentido figurado o indirecto de los mensajes (en contraste con uno literal o directo); inexperticia en generalizar un aprendizaje obtenido en un ambiente, hacia otro/s ambiente/s; déficit en la memoria de trabajo (mantener distintos tipos de información por un lapso breve de tiempo, al mismo tiempo en que se va usando) (Riviere 1997; Feinstein 2010).

Sumado a la dificultad motriz que muchas personas con autismo presentan, también está la sensibilidad anormal (en mayor-hiper- o menor-hipo-grado), en uno o más sentidos (tacto, visión, oído, gusto, olfato); y, más compartido con personas por fuera del espectro autista, las rabietas $\mathrm{y}$ agresiones, autolesiones, ansiedad, tics, hiperactividad, problemas de alimentación, sueño, control de esfínteres y de autocuidado; muchas de éstas, como consecuencia de los déficits comunicativos, cognitivos y/o sensoriales (Riviere 1997; Feinstein 2010).

En fin, las habilidades que están tanto en déficit como en exceso en el espectro autista, y que son susceptibles de aumentar, mejorar y/o ajustar a través de un tratamiento, una vez hecho un diagnóstico exhaustivo, son variadas y pueden alcanzar un número considerable. En la actualidad ha habido un aumento en la producción científica para la identificación de las causas del TEA (Nemirovsky et al, 2015) así como recopilaciones sobre la evolución y desarrollo de terapéuticas (Riviere 1997, Baron-Cohen 2008; Feinstein 2010).

La consideración de un espectro ayuda a dejar de lado la necesidad imperiosa de clasificar a una persona dentro de un subgrupo del mismo, que, por otra parte, puede evolucionar hacia otro con el paso del tiempo, y que, además, comúnmente manifiesta características de otros. Si bien cierto grupo de rasgos es más probable que otro, las fronteras son difíciles de definir. Lo importante, desde el punto de vista clínico, es poder diagnosticar si esa persona se encuentra dentro del espectro, para posteriormente evaluar detalladamente su propio patrón de capacidades (Wing, 1996). Y es aquí donde los tratamientos con apoyo empírico se insertan con éxito.

\section{Terapias actuales para el espectro autista}

Actualmente, las terapias que cuentan con mayor validez debido a su eficacia clínica en el espectro autista, son la terapia ABA (Applied Behavioral Analysis, Leaf y McEachin, 1998; Trivisonno, 2007) y la terapia TEACCH (Treatment and Education of Autistic related Communication Handicapped Children)(Mesibov y Howley, 2003; Mesibov, Shea y 
Schopler, 2006; Callahan, Shukla-Mehta, Magee, y Wie, 2010; Virués-Ortega, 2010).

La terapia $\mathrm{ABA}$ tiene como método principal de enseñanza de habilidades (cognitivas, lingüísticas, académicas, motrices, de conducta, sociales, de juego), el aprendizaje estructurado según tareas discriminadas. Esto incluye: dividir la habilidad en pequeñas partes, enseñar una parte por vez con un inicio marcado por un estímulo (un tipo de instrucción, un modelo, instigación física o verbal, señales), y un final representado por un reforzador (que, entre otras cosas, puede ser diferencial según el desempeño de la parte de la habilidad; por ej: si se logra con instigación, se refuerza de una manera, y si se logra de manera autónoma, de otra), o su ausencia (más informe de que la respuesta fue incorrecta, o faltó), en caso de no darse la respuesta esperada.

Una condición fundamental es que no se puede pasar a la segunda parte de la habilidad si no está lograda totalmente la primera, teniendo en cuenta un criterio de dominio previamente acordado (por lo general, cuando la sub-habilidad se realiza de manera correcta e independiente durante algunos días seguidos). Esto contiene como paso intermedio y según el caso, la existencia de refuerzo por aproximación a la parte de la habilidad requerida (o por moldeamiento), es decir, hacia una respuesta similar, en distintos grados y tras previo acuerdo, a la que se pretende lograr antes de pasar a la segunda.

Una segunda condición es la intercalación con actividades recreativas, libres, o usadas para aprovechar el entrenamiento de ciertas habilidades (aumentar el juego colaborativo, aumentar el uso funcional de objetos, disminuir las rabietas), sin la estructura que imponen las tareas discriminativas. No se pretende mecanizar el aprendizaje, por el contrario, se lo intenta naturalizar lo más posible y plasmarlo de actividades motivantes, sin dejar de lado que las personas con TEA, debido a su condición cerebral y de procesamiento de información, necesitan de mayor estructura de aprendizaje y varias repeticiones al entrenar una habilidad de cualquier tipo, a diferencia de un aprendizaje de tareas continuas, común en el sistema de educación formal, y de aprendizaje más pasivo, incidental y verbal, que les basta, de cierto modo, a las personas sin TEA (Riveros y Mendoza, 2005).

Una tercera condición consiste en ir logrando gradualmente la generalización del aprendizaje de las distintas habilidades, lo que requiere una flexibilización de la estructura. Esto se obtiene abarcando distintos frentes. Primero, la búsqueda de desvanecer los estímulos (instigaciones, modelos, etc.) y a la vez, su modificación (instrucciones más complejas y variadas; hechas por distintas personas). En segunda instancia, trocando los reforzadores, tanto su tipo (artificiales por naturales; es decir, reforzar más socialmente o autorreforzarse) como el programa del que dependen (de razón o intervalo, fijo a variable; es decir reforzar artificialmente cada vez menos), generándose un aprendizaje incidental más que estructurado. Por último, cambiándose a otros ambientes de enseñanza, lo más naturales posibles (casa, colegio, taller), lo que conlleva más adelante que haya otras personas que la lleven a cabo (padres, parientes, profesores, compañeros).

La terapia TEACCH, por su parte, también tiene como objetivo mejorar o potenciar distintas habilidades ya sea en déficit o en nivel superior de personas con TEA, pero el método está más orientado al ambiente educacional, grupal, y prestando mayor importancia a los estímulos/antecedentes (significado/sentido) de la habilidad a desarrollar más que a los refuerzos/motivaciones de la misma; si bien siguiendo, igual que el ABA, el aprendizaje estructurado. Teniendo en cuenta las características generales del TEA, y de cada persona tras ser evaluada, procede, entre otras cosas, mediante: Ambiente estructurado y predecible, para entrenar cada habilidad y el conjunto de ellas, ya sea individual o grupalmente (con zonas como "de actividades individuales", "de juego", "de actividades grupales", "de comida", " de distracción/ relajación", entre otras); dividir cada habilidad a ser aprendida, "en pasos"; uso de estímulos claros, concretos y lo menos ambiguos posibles para la aparición y entrenamiento de la habilidad, y refuerzos motivantes, con apoyo visual en casi todos los casos, en contraste con el verbal, que no basta para el procesamiento autista, e incluso es contraproducente en varios casos; complemento con el aprendizaje incidental (como opuesto al estructurado); fases de relajación, recreo, ejercicio (en los ambientes destinados a esto), combinadas con el aprendizaje de habilidades, incluidas también en una estructura; uso de técnicas como moldeamiento (reforzar aproximaciones de la conducta deseada, hasta hacerlo únicamente con la final) y encadenamiento (hacia adelante -comenzando por la primera parte de una conducta compleja, e ir brindando más ayuda hasta llegar a la final- o hacia atrásempezando por la de mayor complejidad con total ayuda, $\mathrm{y}$ reduciéndola hasta llegar a la primera parte-); anticipación de cada respuesta o secuencia de respuestas exigida, tiempo para hacerla, en qué caso finaliza y qué se obtiene al final (todo con apoyo visual); enseñar el aprendizaje de patrones en cada ambiente, y en conjuntos de ellos (generalización), es decir, que a cada estímulo le debe seguir cierta respuesta dada por el sujeto, y esta será reforzada si se ejerce, u ocurrirá otra consecuencia si no se ejerce (relación de contingencia) con la 
ausencia progresiva de un supervisor/incitador (el aprendizaje de patrones aumenta la probabilidad de darle orden y sentido a los ambientes y personas); la comunicación (expresiva o receptiva) a enseñar debe adaptarse a cada persona, pudiendo trazarse como objetivo el producir un sonido, palabra, frase o gesto, apretar un botón, indicar una palabra o dibujo, o intercambiar un objeto, para pedir algo o para cualquier otra intención comunicativa, y lo mismo ocurre al darle un mensaje a comprender; si esta capacidad está más avanzada, se puede enseñar nuevo vocabulario, morfología compleja, comprensión de códigos sociales compartidos, iniciativa, turnos, solución de problemas, lenguaje escrito, entre otros; el objetivo con demás habilidades como las sociales también puede ir de menor a mayor cualidad, como la proximidad con una persona o grupo, el mirar a otro, responder a un pedido de otro, compartir algo con iniciativa o no, turnarse y seguir reglas de un juego, según la capacidad y potencial de cada uno, y siempre mediante pasos pequeños graduales (primero una breve proximidad con el grupo, cantando una canción o usando un objeto, luego con actividades de comprensión de lenguaje -con apoyo visual-, y finalmente con conversación entre todos, con exigencia de mayor tiempo en el grupo); la evaluación de la comunicación se realiza mediante tres parámetros: forma (a través de una rabieta, objetos, dibujos, palabras en papel, señas, lenguaje escrito, lenguaje verbal), contexto (ambiente y personas) y función (petición, negación, comentar, buscar atención, rutinas sociales), para luego desarrollarla según la potencialidad de la persona; flexibilizar las rutinas de la persona, negociando y de manera gradual, por ejemplo, cambiando de materiales, instigaciones y ambientes, para ir logrando la generalización de los patrones de cada habilidad.

Ambas terapias tienen como objetivo fuerte el generalizar el aprendizaje, para depender menos de los estímulos que estuvieron presentes al momento de adquirirlo y entrenarlo, y así aplicarlo en otros, de manera autónoma.

Según Sundberg y Michael (2001) el foco del tratamiento debe estar en desarrollar la habilidad del lenguaje y comunicación, considerándolo el principal objetivo de la terapia para el autismo, ya que subyace a todas las demás (relaciones sociales, imaginación, síntesis y planificación, habilidades más avanzadas de comunicación como comprensión de códigos sociales, entre otras). Los trabajos de Lovaas (1977) y Taylor y McDonough (1996) han ido en la línea de entrenar lenguaje, principalmente, en personas con TEA, cuyos procedimientos se usan actualmente en las terapias ABA y TEACCH.
Para este logro, que además debe generalizarse, los dispositivos tecnológicos que sirvan de estímulos (visuales, auditivos), recordatorios de respuestas o relaciones de contingencia, respuestas mismas, refuerzos y/o organización/ estructura en general, pueden ser de gran ayuda para estimular la comunicación en niños con TEA.

\section{Objetivos de comunicación en las terapias en TEA y dispositivos tecnológicos de apoyo}

Los objetivos a lograr dentro del espectro autista, en cuanto a las habilidades en general, y a la comunicación y lenguaje en particular, varían dependiendo de la evaluación hecha en un primer paso con determinada persona, respecto a su campo expresivo (uso) como receptivo (comprensión).

Skinner (1957), plantea como unidad de análisis la “operante verbal", con seis tipos según la relación funcional con los estímulos y refuerzos que presente. Esa clasificación ha sido seleccionada por las dos terapias analizadas anteriormente.

Si bien no de manera tan técnica y explícita (Michael, 1984), los objetivos comunicativos, entre muchos otros, pueden ser los siguientes:

- Aumentar, en cuanto a cantidad y/o calidad, el tipo de unidad verbal (u operante verbal) "mando" (unidad controlada por lo que la persona quiere/le falta, y reforzada por eso que se pretende), por ejemplo, decir "puerta" cuando quiere salir de la habitación, lo que es reforzado por la apertura de la misma, o "alto" cuando quiere que bajen el ruido, reforzado por el freno de la aversión.

- Aumentar la operante verbal "ecoico" (o imitación), controlada por el estímulo representado por la misma palabra o frase que se pide repetir, y reforzada por aprobación, o también por una información o un objeto, según el caso (por ej. se hace repetir “ ¿dónde está mi juguete”, detectando el momento en que lo necesita, y se refuerza con "encima de la mesa" y el posterior encuentro con el juguete).

- Aumentar la operante verbal "intraverbal". A diferencia de "ecoico", el estímulo que la antecede no es igual a la respuesta que se espera. Por ejemplo, entrenar decir "perro" y más adelante, "gato", "pato", "león", etc. al ver o escuchar la palabra "animal" corresponde a una intraverbal. Si se le enseñara a la persona a decir "perro" luego de decirle "perro", sería un ecoico. Por otro lado, si se le enseñara a decir "perro" al ver el dibujo de un perro, o uno real, sería una operante "tacto". Y por último, si se le hiciera desarrollar la palabra "perro" al verla escrita, correspondería a una operante "textual". Para resaltar la importancia de 
la operante intraverbal, así como considerándola de un nivel más avanzado e ideal (a diferencia del nivel básico del "mando"), la misma permite poseer lenguaje sobre objetos o eventos no presentes (lograr relaciones o redes de palabras/conceptos mentales), a diferencia del "tacto".

- Sólo después de que la persona tiene en su repertorio las operantes verbales arriba descritas, puede pasarse a objetivos de conductas lingüísticas más complejas (Sundberg y Michael, 2001; Juillerat et al., 2015). Por ejemplo, comprensión y expresión de reglas gramaticales como la estructura sujeto-verbo-objeto ("El niño toca al perro"), preguntas con respuestas "sí-no" (“¿Es esto un perro?"), preguntas abiertas (“¿Qué hiciste hoy?”); conceptos (arriba, dentro, alto, bajo, ayer, hoy), entre otros.

- El lenguaje receptivo (o comprensión, como lo efectuado mediante el sistema PECS, Picture Exchange Communication System de Frost y Bondi, 1994 si bien es necesario y ha demostrado eficacia (Tien, 2008), no es suficiente para el entrenamiento según la perspectiva de Skinner, ya que la persona aprende a apuntar el dibujo de lo que se le pregunta, pero no siempre a usar esto como comunicación funcional en el momento en que necesita tal objeto o información, es por eso que deben detectarse los estímulos correctos que den lugar al uso del sistema, y además privilegiar el lenguaje expresivo. Sin embargo, en algunos casos debido al bajo potencial de aprendizaje, el objetivo debe quedar en este nivel.

Por otro lado, según los objetivos propuestos por Lovaas (1981) en cuanto a habilidades de lenguaje, éstos deben avanzar de manera gradual desde imitación de acciones simples ("Haz esto" -aplaudir-), pasando por imitación de acciones con objetos ("Haz esto"-chocar dos cubos-), primer lenguaje receptivo (pedirle "Aplaude" o "Cierra la puerta"), imitación verbal (congruente con lo visto más arriba de Skinner; en este caso, comenzando por cualquier vocalización -"aaa", "jaja"-, después de decirle "Habla"; luego desarrollando imitación de sonidos específicos, "Di baa"; e imitación de palabras, "Di banana"), designación expresiva de objetos (Mostrar un libro y decirle "¿Qué es esto?”, o mostrar varias imágenes y decirle “¿Qué quieres?”), designación expresiva de acciones (saltar y decirle "¿Qué estoy haciendo?"), designación receptiva de objetos (mostrar una taza y pedirle "Toca la taza"), y designación receptiva de acciones (decirle "Camina hacia la cama" o "Señala caminar/comer" -en dibujos-); hasta terminar con lenguaje avanzado (describir atributos de objetos -tamaño, color y forma-, relaciones entre objetos -debajo, dentro-, usar pronombres posesivos que indican relación entre el objeto y la persona -tuyo, mío-, conceptos abstractos como tiempos -ayer, hoy, primero, último-, respuestas sí/no, y estructura de frases más largas "Quiero comida").

Frente a todas estas necesarias posibilidades de tratamiento, los dispositivos tecnológicos usados para apoyar el aprendizaje durante él, y posteriormente, para generalizarlo, con la mayor autonomía posible de la persona, pueden funcionar como un gran auxiliar de la psicoterapia.

\section{Tecnología y TEA}

Desde hace décadas, se ha indagado sobre la relevancia de la tecnología aplicada en la terapia y en la clínica en TEA. Particularmente, las acepciones de la tecnología implican una aplicación práctica del conocimiento sobre un determinado campo, comprendiendo cada elemento u objeto utilizado en un campo de estudio, dependiendo del contexto sociohistórico en el cual está enmarcado (Quintanilla, 1989; Osorio, 2002).

Las necesidades tecnológicas en la vida diaria no se corresponden directamente con las necesidades cognoscitivas. Sin embargo, cuando existen mayores dificultades, es necesario pensar en la utilización responsable y dirigida de objetos o elementos que ayuden al desarrollo tanto práctico como social de las personas. La disposición de elementos tales como telefonía móvil, dispositivos digitales táctiles, interfaces virtuales personales y comunitarias, abre el campo de interacción y de respuesta a un universo casi ilimitado de posibilidades en el cual es fácilmente posible perderse en la creencia de que la tecnología. Asi, usar tecnologías que pueden no tener un uso específico construyen una red de elementos que juntas, y con la dirección motivacional adecuada, dan lugar a nuevas y mejores estrategias terapéuticas y de educación para personas con TEA así como también llegar a ellos desde los vínculos familiares y terapéuticos. (Goldsmith y LeBlanc, 2004).

\section{Proceso de comunicación mediado por asistencia}

Un acercamiento para tratar de establecer un puente entre la persona con TEA y su mundo cercano, radica en buscar estrategias mediadas por sistemas de comunicación aumentada y alternativa (AAC). Se denomina así a la implementación de tecnologías surgidas de la práctica clínica terapéutica como herramientas para ayudar a resolver los retos de comunicación que muchas personas con TEA experimentan, ayudando a compensar esas deficiencias. (American Speech-Language- 
Hearing Association, 1997; Glennen 1997)

En los últimos años, los AAC se nutren de las tecnologías digitales (computadoras, celulares, tabletas digitales, etc) con softwares que disponen de múltiples funciones, accesibles y tienen rápida acción/reacción, permitiendo que la barrera de las dificultades comunicacionales sea menos rígida (Hetzroni y Tannous, 2004); esto conforma el dispositivo AAC propiamente dicho (Lubas, Mitchell y De Leo, 2014).

Existen varias revisiones que han demostrado que la utilización del sistema de comunicación por intercambio de imágenes (PECS), mejoran las capacidades de comunicación en los niños con TEA (Flippin, Reszka y Watson 2010; Ganz, Simpson y Lund 2012; Ganz, Hong y Goodwyn, 2013). Este sistema se basa en una estructura de pasos que, a través de imágenes conceptuales de acciones/emociones y de objetos cotidianos, la persona con TEA puede, progresivamente, construir una vía de comunicación.

A pesar de los beneficios de PECS, hay barreras logísticas de implementación que pueden mejorarse para hacer este sistema más conveniente, útil y eficaz, utilizando la colaboración de los avances tecnológicos. Los materiales que lo conforman, con el tiempo se deterioran, aunque no lo hacen conceptualmente en la persona con TEA, con lo cual, la diferencia entre el elemento real y el conceptual genera situaciones de estrés. Esto, a su vez, no sucede con medios virtuales que son imperturbables. Sin embargo, el mismo método de comunicación por intercambio, se basa, precisamente, en el intercambio físico de un objeto con lo que lleva todo un proceso que es reemplazado en la tecnología por un elemento intangible, virtual y reduccionista.

Es importante comprender en qué aspectos, en conjunto e individualmente, los entornos virtuales en las modalidades de comunicación facilitadas son adecuados de acuerdo a: tipo de representación que se utiliza (por ejemplo, fotos reales vs caricaturesco como pictogramas), representación física (de dos o tres dimensiones), su capacidad de adaptación, el control que el usuario tiene sobre ellos, y su portabilidad (Wass y Porayska-Pomsta, 2014).

Un ejemplo de esto son los organigramas: maquetas que utilizan pictogramas puestos en sucesión para indicar una secuencia temporal de acciones o actividades futuras o realizadas. Son necesarios para los niños que en la gran mayoría tenga problemas con la memoria secuencial y organización del tiempo. Este tipo de elementos le ayuda a la persona a predecir acontecimientos y trae como consecuencia un menor nivel de ansiedad y mayor tranquilidad ya que sabe/sabría cuál es la próxima actividad a realizar. Si fuera maquetado en físico, utilizando pictogramas de cartón o a través de un contador digital, los resultados deberían ser los mismos, pero la interpretación del acontecimiento por la representación del modelo en sí, es diferente. Por eso, la selección del modo de presentación de la tarea es un elemento clave que debe ser orientado hacia las necesidades e intereses de la persona. Niños con baja iniciativa se ven más motivados a completar una tarea que no es de su agrado si al mirar su cronograma al finalizar esta tarea seguirá una que sí es de su agrado (Fletcher-Watson, Hammond y O'Hare, 2013).

Es importante que la tecnología sea personalizable para evitar también un desapego por parte de los usuarios aún si la idea sigue consistiendo en fomentar desarrollo a través de una herramienta que se quiera y no como una obligación impuesta. Los motivadores son variados, dependiendo de cada una de las personas. Cargar una gran cantidad de imágenes propias de la persona y el diseño de lo que se ve tiene que ser acorde, por lo cual la personalización abastece las necesidades del momento (Fortalezas, Intereses y Dificultades de la persona) y genera un entorno confiable para la persona.

Así, almacenar material audiovisual y vincularlo al contexto en el cual el niño con TEA se desenvuelve de la manera que considere más oportuna, deben estar bien definidos, identificados y ubicados para que el niño los pueda manipular cuando sea el momento oportuno y los encuentre de manera acorde a él, donde la intervención en el desarrollo no sólo debe descansar en las cuestiones técnicas sino también poder involucrar al entorno familiar de la persona, siempre y cuando sea esto posible (Porayska-Pomsta et al., 2013; Wass y Porayska-Pomsta, 2014).

En un estudio de efectividad de tecnologías asistenciales en autismo, un 33\% retuvo mejor la información si ésta era presentada a través de computadoras o tabletas, que siendo instruidas por una maestra asistencial (McEwen, 2014). También, la implementación de tabletas digitales mejoró un $25 \%$ la habilidad de comunicación y lenguaje social en 61 pacientes con TEA, que sin la intervención de esta tecnología en actividades relacionadas (Tager-Flusberg, 2014). Tener recursos digitales ayuda pero no excluye el crecimiento del conocimiento. Se entiende también que abre un mundo de posibilidades en el aula para poder desarrollar otras habilidades en déficit. Esta tecnología puede usarse para trabajar la atención, anticipación, funciones ejecutivas en general, memoria de trabajo, secuencias de acciones, organización de eventos, como agenda, y cronogramas. El desarrollo tecnológico permite innovaciones constantes que incluyan la posibilidad de iniciar, mantener o terminar 
comportamientos; ayudar en el desarrollo de habilidades de seguridad, imitación vocal, participación grupal, atención a los detalles y generalización, organizar de manera cuidada y directa los eventos y situaciones difíciles.

Pensando hacia adelante es clave entender que el mundo real es multisensorial, con lo cual las diferencias limitadas de expresión del mundo digital actual tiene que tender a cerrarse hacia experiencias más reales, que sean seguras y adaptadas para lograr beneficios más específicos y eficaces.

\section{Otras tecnologías en asistencia comunicacional: Robótica y entornos virtuales}

La aplicación de tecnologías industriales y de multimedia como la robótica y los entornos virtuales, al TEA, es un recurso explotado actualmente. Es una posibilidad real que den frutos si se aplican en contextos para el manejo de situaciones ansiogénicas o de sobrecarga emocional, considerando también las cuestiones éticas basadas no solo en la terapia acordada con los profesionales de la salud sino también en las necesidades de la persona.

Los estudios que se han realizado en este campo tienden a adoptar la forma de interacción y/o imitación, es decir por ejemplo, los robots que copian los movimientos, juguetes y peluches amigables que responden con estímulos positivos o de socialización, varían según su morfología y aproximación para cada persona (Coeckelbergh et al. 2016). La interacción con los robots tiende a ser predecible y repetitiva, que son el tipo de interacciones que algunos individuos con TEA prefieren. Además, permite obtener datos de interacción y ayudar a que las personas puedan tener un vínculo con una interfaz que no le genere un estrés emocional o social (Dautenhahn y Werry, 2004). Controlar robots amigables abre una ventana de vínculo social controlado, con tareas simples y concretas, de respuesta rápida y sencillas. El factor vinculante entre las personas y este tipo de interfaz es un estudio interesante para promover complementariamente con las situaciones sociales y familiares propias de la persona con TEA (Scassellati, Admoni y Mataric, 2012; Kozima, Nakagawa y Yasuda, 2005; Coeckelbergh et al. 2016; Dautenhahn y Werry, 2004).

Los entornos de realidad virtual ofrecen la posibilidad de presentar las tareas de aprendizaje de forma que sean realistas pero dentro de un entorno simplificado. Estas interfaces van desde entornos tridimensionales que utilizan una palanca de mando y un computadora personal (cascos de realidad virtual para terapeutica de fobias o recorrer lugares prediseñados visto en Rojas, Tarnogol y Yang, 2016) hasta entornos totalmente inmersivos sin una pantalla, estrictamente en ambientes virtuales con representación en toda la habitación controlado por el cuerpo del niño con TEA y tutores (Herrera, Jordan y Vera, 2006; Vera, Campos, Herrera y Romero, 2007; Herrera, Alcantud, Jordan, Blanquer, Labajo y De Pablo, 2008). Proyectos que utilizan proyectores y sensores de profundidad que permiten construir y darle al usuario una capacidad propia como también guiada por un tutor en entornos amigables que no se restrinjan a un objeto táctil, teniendo que utilizar el cuerpo para poder interactuar, y que emulan movimientos naturales de interacción como señalar, saltar, sentarse y trabajar el juego simbólico (Herrera et al., 2012).

\section{Consideraciones de la tecnología en la terapéutica del TEA}

Hablando de acortar la distancia en la brecha digital/real, la simplificación de elementos de la realidad ofrece una paradoja. El desarrollo del aprendizaje y la comprensión de elementos complejos en la realidad es una dificultad que con la reducción digital, tardaría más en desarrollarse. Sin embargo, este ambiente reducido evita la sobrecarga sensorial logrando que sea un entorno atractivo y de confort para personas con TEA.

La ventaja, sin embargo, es que al ser repetitivo, no genera situaciones inesperadas que provocan estrés mediante respuestas inmediatas y predecibles, frente a demandas sociales en comunicación con el otro o en un grupo social, de la vida real; tampoco genera prejuicios, no juzga ni genera un entorno de conflicto en los niños. Los programas específicos en entornos educativos o de aprendizaje terapéutico, son consistentes en la definición de tareas definidas, lo cual reduce las distracciones por estímulos sensoriales innecesarios y focaliza la atención en el desarrollo de las actividades, generando también un entorno con ayudas visuales y atractivas tanto para el niño como para los terapeutas (Porayska-Pomsta et al., 2013; Wass y Porayska-Pomsta, 2014).

Es importante remarcar el papel fundamental del vínculo del profesional de la salud con el niño. El corolario es que el uso apropiado de las nuevas tecnologías puede ser un apoyo para la educación y para el tratamiento del TEA, siempre que sea consciente de cuándo retirar el apoyo (Kandalaft, Didehbani, Krawczyk, Allen y Chapman, 2013; Kozima, Yasuda y Nakagawa, 2007; Feil-Seifer y Mataric, 2008; Wass, Scerif y Johnson, 2012). La tecnología por sí sola no ayuda, por eso tiene que estar mediada por personas para 
lograr vincularidad, pero en la actualidad hay un fácil acceso a ella ofreciendo también una oportunidad de desbalancear el acercamiento y la estabilidad del desarrollo de las terapias en el consultorio. Las tecnologías pueden sólo complementar pero nunca puede sustituir el tipo cuidado humano y el apoyo social y terapéutico (Cahill, Macijauskiene, Nygård, Faulkner y Hagen, 2007). Es una cuestión clave a la hora de lograr una inserción exitosa considerar también la importancia de poder reducir los costos de aprendizaje a largo plazo.

\section{Generalidades de un Proyecto de Tecnología Aplicada: Investigación y desarrollo}

La primer etapa de una investigación aplicada en ciencias cognitivas, en general, y en TEA en particular, consiste en armar una herramienta o dispositivo acorde. Este debe ser intuitivo, cumplir con las principales características y necesidades que busquen tanto los padres como terapeutas de personas con TEA. Además debe ser personalizable, focalizado en la persona, y ofrecer capacidad de generar estímulos visuales y auditivos. Luego, el niño con TEA es entrenado por el terapeuta con el fin de comenzar a comunicarse con el mundo exterior y a tomar decisiones con opciones planteadas utilizando la herramienta, motivando una característica comunicacional.

El proyecto Apptismo, particularmente, está inmerso en una investigación en el campo de las neurociencias, más específicamente orientado sobre el área neurocognitiva-social. Esencialmente aborda la utilización de la tecnología para el estudio del desarrollo cognitivo en personas con TEA. De esta manera, se busca generar herramientas económicas, accesibles, inclusivas, integradoras e innovadoras que motiven la expresividad y mejoren la calidad de vida del niño, brindando además un nueva vía terapéutica. Utilizando software para celulares, dispositivos digitales táctiles y relojes inteligentes se puede abarcar una amplia gama de experiencias de usuario que contribuyen al espectro de datos posibles de recabar para lograr una investigación robusta.

El objetivo de la investigación es medir el desarrollo cognitivo en pacientes con TEA; de esta forma se determina qué puntos pueden ser optimizados para obtener mejores resultados y lograr así que la persona incremente significativamente sus habilidades comunicacionales. Esta es una oportunidad para la investigación planteada en un contexto más cercano a las ciencias psico-sociales partiendo desde la ciencias computacionales, siendo esta relación de integración y multidisciplina, con las cuales se pueden obtener resultados mensurables en el comportamiento y desempeño cognitivo en las personas durante el estudio.

El uso de Apptismo podría extenderse a otros niveles de severidad e incluso para el tratamiento terapéutico de otras enfermedades. Utilizando el comunicador o el organizador de Apptismo para pacientes con secuelas de Accidentes Cerebro-Vasculares, Alzheimer u otras patologías de la memoria (Bravo et al., 2008), se puede plasmar "el mundo" en una pantalla, una frase difícil de expresar a pesar de poder comprender la situación o el contexto o el mismo entrenamiento de la motricidad fina perdida.

Considerar que el uso es plenamente positivo o negativo es una de las principales causas de resistencia, y por consecuencia, impedimentos en la innovación tecnológica y terapéutica. Es clave entender que las aplicaciones tecnológicas, previas a su uso, deben estar bajo una rigurosa evaluación dentro de un contexto académico y que tenga evidencia comprobada suficiente, tanto académica como terapéutica (Fletcher-Watson, 2015). De esta manera, podemos tener un contexto más seguro y ordenado de las aplicaciones, evitar conflictos y problemas que den como resultado la separación de herramientas potencialmente beneficiosas y generar posibilidades a nuevos desarrollos futuros basados en la evidencia comprobada.

Todavía quedan grandes posibilidades para el desarrollo cognitivo utilizando las nuevas tecnologías, el soporte interdisciplinario y el aporte de una investigación que permita cuantificar los resultados

\section{Características del Proyecto Apptismo}

El objetivo del proyecto, es que además de diseñar y planificar un software, es que sea inmerso en una investigación que evalúe la eficacia de Apptismo como una herramienta para estimular habilidades comunicacionales, organizacionales o en déficit específicas. En cuanto al funcionamiento de la aplicación, se pueden destacar dos modos de uso: el "modo usuario" destinado al paciente, que tendrá categorías y sus correspondientes subcategorías de pictogramas, los cuales representarán situaciones y características del mundo real de interés para el entrenamiento del paciente; y el "modo edición" destinado a terapeutas, padres o tutores, quienes administrarán el material audiovisual que utilicen la herramientas, pudiendo modificar el número de categorías, los textos, los colores, los sonidos y los videos considerados apropiados para generar un perfil adecuado para cada situación. Muchos productos/ aplicaciones específicas que cumplen con las necesidades de personas con TEA no pueden cuantificar resultados o dar 
una idea de verdadero desarrollo de la persona, lo cual no la difiere de un juguete de gusto por el momento. Por otro lado, hay que considerar el hardware y el sistema operativo sobre el cual funciona la aplicación para dar lugar a un mayor acceso y la posibilidad de llegar a más personas en contextos complejos económica y socialmente. Apptismo está desarrollado para tabletas digitales táctiles, principalmente, de sistema operativo Android, para tamaños de 7 y 10 pulgadas, con descarga gratuita. Su estructura permite incorporar imágenes, videos y personalizar hasta 64 pictogramas (con texto, color y música) según las necesidades del momento, para ser intercambiadas ante eventualidades inesperadas. Es compatible con los pictogramas de ARASAAC (www. arasaac.org). Así mismo, registra el uso en tiempo y cantidad de veces que se elige cada categoría y subcategoría por parte del usuario con la finalidad de captar la eficiencia y efectividad a través de análisis estadísticos posteriores. Esto da lugar no solo a una gran cantidad de datos que den luz sobre cómo ejecutar las mejoras sino también, discernir entre intereses y deficiencias en las personas con TEA y también mejorar las actividades por parte del grupo terapéutico.

El proyecto se realiza en el Laboratorio de Aprendizaje Biológico y Artificial, dentro del Departamento de Ingeniería Biomédica del Instituto de Ciencias Básicas y Medicina Experimental del Instituto Universitario del Hospital Italiano. La investigación cuenta con la aprobación del comité de ética del Hospital Italiano, consentimiento de los individuos involucrados y la participación del servicio de Salud Mental Pedátrica del Hospital Italiano de Buenos Aires. La evaluación se realiza en consultorios del servicio donde se aplican las terapias acordes a cada persona en un grupo multidisciplinario, siempre bajo la supervisión del terapeuta y/o médico.

Abarcar todos los niveles de déficit en todos las deficiencias en un trastorno del neurodesarrollo para una única aplicación la lleva cerca de la inviabilidad, por lo que debemos recurrir a una constelación de aplicaciones. Lograr esto es nuestro objetivo, considerando que se requieren múltiples intervenciones para el diseño y el desarrollo cómo integrar diferentes disciplinas (psicología, psiquiatría, diseño grafico, ingenieria, neurociencias, etc ) para el análisis de resultados y el planteo del uso de la herramienta. En particular, Apptismo funciona como un introductor, lo que quiere decir que es la puerta de entrada hacia la adaptación de los individuos a otras aplicaciones dentro de la constelaciòn para trabajar aspectos más específicos con mayor facilidad.

\section{Conclusión}

La elección de las estrategias (centrándose en una visión a largo plazo), debe estar relacionada con el estudio de los niveles actuales del funcionamiento del individuo y sus fortalezas y debilidades. Ante la severidad y cronicidad del autismo, no es sorprendente que muchas alternativas no debidamente justificadas se propongan con beneficios esperanzadores.

A causa de que muchas disciplinas están implicadas en los aspectos de la evaluación y el tratamiento, resulta importante que el profesional de salud esté en principio involucrado con los padres en el desarrollo del plan de cuidados para el niño. Así mismo los padres deben considerar pedir ayuda cuando sea necesario para tomar las decisiones más acertadas y reales. Lo mejor es presentar una relación terapéutica con la menor restricción posible para la persona con TEA, junto a la mayor eficacia y la mayor seguridad posible.Además, para determinar si efectivamente una aplicación tecnológica influirá en dicho aspecto, es fundamental una investigación que la respalde, basado en la evidencia. Por esta razón, se propone desarrollar aplicaciones en un contexto donde se medirán los resultados obtenidos en personas y se podrá determinar cuán efectiva es la misma y qué características son las que más se deben tener en cuenta para mejorar la inserción y su desarrollo. El objetivo del uso de dispositivos tecnológicos es facilitar aprendizajes de manera más eficiente y efectiva, que a su vez, generen mayor aceptación en el uso para el niño con TEA. Por lo tanto, es relevante implementar el uso de estas plataformas para que posibilite un objetivo terapéutico claro, facilitando el trabajo con sus terapeutas o en las actividades diarias con sus padres. 


\section{Referencias}

American Psychiatric Association. (1994). Diagnostic and Statistical Manual for Mental Disorders: DSM-IV (Fourth Edition). Washington, DC: American Psychiatric Publishing.

American Psychiatric Association. (2013). Diagnostic and statistical manual of mental health disorders: DSM-5 (Fifth Edition). Washington, DC: American Psychiatric Publishing.

American Speech-Language-Hearing Association. (1997). Augmentative and alternative communication (AAC)._http://www.asha.org/public/ speech/disorders/AAC.htm

Asperger, H. (1944). Die autistischen Psychopathen im kindersalter. Archiv für Psychiatrie und Nervenkrankheiten, 117, 76-136.

Baron-Cohen S. (2001). Theory of mind in normal development and autism. Prisme. 34, 174-183.

Baron-Cohen S. (2008). Autism and Asperger Syndrome. Estados Unidos: Oxford University Press.

Bravo, J., López-De-Ipiña, D., Fuentes, C., Hervás, R., Peña, R., Vergara, M., y Casero, G. (2008). Enabling NFC technology for supporting chronic diseases: A proposal for alzheimer caregivers. In Ambient Intelligence (pp. 109-125). Springer Berlin Heidelberg.

Cahill, S., Macijauskiene, J., Nygård, A. M., Faulkner, J. P., y Hagen, I. (2007). Technology in dementia care. Technology and Disability, 19, 55-60.

Callahan, K., Shukla-Mehta, S., Magee, S., y Wie, M. (2010). ABA versus TEACCH: the case for defining and validating comprehensive treatment models in autism. Journal of autism and developmental disorders, 40, 74-88.

Coeckelbergh, M., Pop, C., Simut, R., Peca, A., Pintea, S., David, D., y Vanderborght, B. (2016). A survey of expectations about the role of robots in robot-assisted therapy for children with ASD: Ethical acceptability, trust, sociability, appearance, and attachment. Science and engineering ethics, 22, 47-65.

Dautenhahn, K., y Werry, I. (2004). Towards interactive robots in autism therapy: Background, motivation and challenges. Pragmatics y Cognition, 12, 1-35.

Feil-Seifer, D., y Mataric, M. J. (2008, August). B 3 IA: A control architecture for autonomous robot-assisted behavior intervention for children with Autism Spectrum Disorders. In RO-MAN 2008-The 17th IEEE International Symposium on Robot and Human Interactive Communication (pp. 328-333). IEEE.

Feinstein A. (2010). A History of Autism: Conversations with the Pioneers. NJ, Estados Unidos: John Wiley y Sons

Fletcher-Watson, S. (2015). Evidence-based technology design and commercialisation: Recommendations derived from research in education and autism. TechTrends, 59, 84-88.

Fletcher-Watson, S., Hammond, S., y O'Hare, A. (2013). Click-east: evaluating the impact of an ipad app on social communicative abilities in young children with autism. IMFAR, San Sebastian, Spain, 4.

Flippin, M., Reszka, S., y Watson, L. R. (2010). Effectiveness of the Picture Exchange Communication System (PECS) on Communication and Speech for Children With Autism Spectrum Disorders: A Meta-Analysis. Am J Speech Lang Pathol, 19, 178-195. doi: 10.1044/1058-0360(2010/09-0022).

Frith, U. (1992). Autismo: hacia una explicación del enigma. Madrid: Alianza Editorial.

Frost, L. A., y Bondy, A. S. (1994). The picture exchange communication system training manual. Cherry Hill, NJ: Pyramid Educational Consultants.

Ganz, J. B., Hong, E. R., y Goodwyn, F. D. (2013). Effectiveness of the PECS Phase III app and choice between the app and traditional PECS among preschoolers with ASD. Research in Autism Spectrum Disorders, 7, 973-983.

Ganz, J. B., Simpson, R. L., y Lund, E. M. (2012). The picture exchange communication system (PECS): A promising method for improving communication skills of learners with autism spectrum disorders. Education and Training in Autism and Developmental Disabilities, 176-186.

Glennen, S. L., y DeCoste, D. C. (1997). The handbook of augmentative and alternative communication. San Diego, CA: Singular.

Goldsmith, T. R., y LeBlanc, L. A. (2004). Use of technology in interventions for children with autism. Journal of Early and Intensive Behavior Intervention, 1, 166.

Herrera, G., Alcantud, F., Jordan, R., Blanquer, A., Labajo, G., y De Pablo, C. (2008). Development of symbolic play through the use of virtual reality tools in children with autistic spectrum disorders Two case studies. Autism, 12, 143-157.

Herrera, G., Casas, X., Sevilla, J., Rosa, L., Pardo, C., Plaza, J., ... y Le Groux, S. (2012). Pictogram Room: Natural Interaction Technologies to Aid in the Development of Children with Autism. Annuary of Clinical and Health Psychology, 8, 39-44.

Herrera, G., Jordan, R., y Vera, L. (2006). Abstract concept and imagination teaching through virtual reality in people with autism spectrum disorders. Technology and Disability, 18, 173-180.

Hetzroni, O. E., y Tannous, J. (2004). Effects of a computer-based intervention program on the communicative functions of children with autism. Journal of autism and developmental disorders, 34, 95-113.

Juillerat, K. L., Cornejo, F. A., Castillo, R. D., y Chaigneau, S. E. (2015). Procesamiento semántico de palabras epistémicas y metafísicas en niños y adolescentes con Trastorno de Espectro Autista (TEA) y con Desarrollo Típico (DT). Terapia psicológica, 33, 221-238.

Kandalaft, M. R., Didehbani, N., Krawczyk, D. C., Allen, T. T., y Chapman, S. B. (2013). Virtual reality social cognition training for young adults with high-functioning autism. Journal of autism and developmental disorders, 43, 34-44.

Kanner, L. (1943). Autistic disturbances of affective contact. Nervous Child, 2, 217-250

Kozima, H., Nakagawa, C., y Yasuda, Y. (2005, August). Interactive robots 
for communication-care: A case-study in autism therapy. In Robot and Human Interactive Communication, 2005. ROMAN 2005. IEEE International Workshop on (pp. 341-346). IEEE.

Kozima, H., Yasuda, Y., y Nakagawa, C. (2007). Social interaction facilitated by a minimally-designed robot: Findings from longitudinal therapeutic practices for autistic children. In RO-MAN 2007-The 16th IEEE International Symposium on Robot and Human Interactive Communication (pp. 599-604). IEEE.

Leaf, R., y McEachin, J. J. (1998). A work in progress: Behavior management strategies and a curriculum for intensive behavioral treatment of autism. New York: Different Roads to Learning.

Lovaas, O. I. (1977). The autistic child: Language development through behavior modification. New York: Irvington.

Lovaas, O. I. (1981). Teaching developmentally disabled children: The ME book. Baltimore: University Park Press.

Lubas, M., Mitchell, J., y De Leo, G. (2014). User-Centered Design and Augmentative and Alternative Communication Apps for Children With Autism Spectrum Disorders. Sage Open, 4.

McEwen, R. (2014). Mediating sociality: the use of iPod Touch ${ }^{\mathrm{TM}}$ devices in the classrooms of students with autism in Canada. Information, Communication y Society, 17, 1264-1279.

McNaughton, D., y Light, J. (2013) The iPad and mobile technology revolution: benefits and challenges for individuals who require augmentative and alternative communication. Augment Altern Commun, 29, 107-116. doi: $10.3109 / 07434618.2013 .784930$

Mesibov, G. B., Shea, V., y Schopler, E. (2006). The TEACCH approach to autism spectrum disorders. New York: Springer

Mesibov, G., y Howley, M. (2003). Accessing the curriculum for pupils with autistic spectrum disorders: using the TEACCH programme to help inclusion. London: David Fulton.

Michael, J. (1984). Verbal behavior. Journal of the Experimental Analysis of Behavior, 42, 363- 376.

Mundy, P., Sigman, M., Ungerer, J., y Sherman, T. (1986). Defining the social deficits of autism: The contribution of non-verbal communication measures. Journal of child psychology and psychiatry, 27, 657-669.

Nemirovsky, S. I., Córdoba, M., Zaiat, J. J., Completa, S. P., Vega, P. A., González-Morón, D., ... y Revale, S. (2015). Whole genome sequencing reveals a de novo SHANK3 mutation in familial autism spectrum disorder. PloS one, 10, e0116358.

Osorio, C. (2002). Enfoques sobre la tecnología. CTS +I: Revista Iberoamericana de Ciencia, Tecnología, Sociedad e Innovación, 7.

Porayska-Pomsta, K., Anderson, K., Bernardini, S., Guldberg, K., Smith, T., Kossivaki, L., ... y Lowe, I. (2013). Building an Intelligent, Authorable Serious Game for Autistic Children and Their Carers. In Advances in Computer Entertainment (pp. 456-475). Springer International Publishing. Quintanilla, M., (1988) Tecnología: Un Enfoque Filosófico, Madrid: Fundesco, 1988.
Riveros, V. S., y Mendoza, M. I. (2005). Bases teóricas para el uso de las TIC en Educación. Encuentro educacional, 12.

Riviere, A. (1997): “Desarrollo Normal y Autismo”. Madrid: Universidad Autónoma de Madrid.

Rojas, F., Tarnogol, F., y Yang, H. S. (2016). Dynamic social formations of pedestrian groups navigating and using public transportation in a virtual city.The Visual Computer, 32(3), 335-345.

Scassellati, B., Admoni, H., y Mataric, M. (2012). Robots for use in autism research. Annual review of biomedical engineering, 14, 275-294.

Skinner, B.F. (1957). Verbal behavior. New York: Appleton-Century-Crofts. Sundberg, M. L., y Michael, J. (2001). The benefits of Skinner's analysis of verbal behavior for children with autism. Behavior Modification, $25,698-724$.

Tager-Flusberg, H. (2014). Promoting Communicative Speech in Minimally Verbal Children With Autism Spectrum Disorder. Journal of the American Academy of Child y Adolescent Psychiatry, 53, 612-613.

Taylor, B. A., y McDonough, K. A. (1996). Selecting language programs. En C. Maurice, G. Green, y S. C. Luce (Eds.), Behavior interventions for young children with autism (pp. 63-177). Austin, TX: Pro Ed.

Tien, K. C. (2008). Effectiveness of the Picture Exchange Communication System as a functional communication intervention for individuals with autism spectrum disorders: A practice-based research synthesis. Education and Training in Developmental Disabilities, 61-76.

Trivisonno, C. (2007). Introducción al enfoque ABA en autismo y retraso de desarrollo: Un manual para padres y educadores. Madrid: Lulu Ediciones.

Vera, L., Campos, R., Herrera, G., y Romero, C. (2007). Computer graphics applications in the education process of people with learning difficulties. Computers y Graphics, 31, 649-658.

Virués-Ortega, J. (2010). Applied behavior analytic intervention for autism in early childhood: Meta-analysis, meta-regression and dose-response meta-analysis of multiple outcomes. Clinical psychology review, 30, 387-399.

Wass, S. V., y Porayska-Pomsta, K. (2014). The uses of cognitive training technologies in the treatment of autism spectrum disorders. Autism, $18,851-871$.

Wass, S. V., Scerif, G., y Johnson, M. H. (2012). Training attentional control and working memory-Is younger, better?. Developmental Review, 32, 360-387.

Wing, L. (1996). The Autistic Spectrum: A Guide for Parents and Professionals. London, UK: Constable and Robinson. 